\section{How did AIDS begin?}

What is to be made of a death from pneumonia in 1959, after an apparent immunological collapse?

A THOROUGHLY compelling tale came to light in last week's issue of The Lancet - that of how it has been possible, by making use of the technique of the polymerase chain reaction (PCR) for amplifying pieces of DNA, to prove that a seaman who died at a Manchester (northwest England) hospital in 1959 most probably died of AIDS (G. Corbitt, A. S. Bailey \& G. Williams, Lancet 336, 51; 1990).

There are two things to say, of which the most obvious is that the report is another vivid attestation of the power of PCR to identify particular nucleotide sequences in tissues long since dead in all clinical senses. On this occasion, by good fortune, some samples of tissue from the dead man had been preserved in blocks of paraffin wax. What now unambiguously emerges is that the kidney, bone marrow, spleen and pharyngeal mucosa (but not samples of liver and brain) carry nucleotide sequences characteristic of the virus believed to be responsible for AIDS, HIV (human immunodeficiency virus). Like Lazarus, it seems, the dead (and their secrets) have been brought to life.

The interest of this development is that it will throw further confusion on questions of the origin of AIDS, of its apparently rapid spread in the past decade and of its long-term potential. The year 1959 is a significant one because it is that from which dates the earliest sample of blood which is known to contain antibodies against HIV, collected in Zaire. So at least two adults died of what would now be called AIDS in 1959. It is unlikely that they were the only victims in that year. And if there were at least two victims in 1959, how many were there in 1958 , and $1957 \ldots$ ?

Nobody, of course, can know these things for certain. But the infection appears to have been much the same in 1959 as it is now. The dead Manchester seaman appears to have infected his wife and she the youngest of their three daughters, both of whom afterwards died of a similar syndrome. For what it is worth, the fifties was also the decade during which immunodeficiency diseases came to the fore under the general heading of 'leukaemia'. There was a ready explanation at the time: that previously, without the benefit of antibiotics, people with immune deficiency such as that associated with leukaemia had died of chance infection. It would be surprising if those deaths did not include a sprinkling of AIDS patients. That would agree with estimates of the time since the divergence of HIV from the simian immunodeficiency viruses from which they appear to be derived (see, for example, T. F. Smith et al. Nature 333, 573; 1988).

If there were sporadic AIDS cases during these years, why did the present spread of AIDS in developed countries not begin until the end of the 1970s? HIV is not especially infectious, has a long incubation time, but eventually kills those infected. The sad case of the Manchester seaman shows how an isolated case of infection may run into the sand, with personal tragedy but no spread of infection beyond the immediate family. Given HIV, the rate at which the infection spreads is determined by the rate at which infected people acquire and infect new sexual partners in the interval between infection and death, with the transfusion of infected blood, the use of infected hypodermic needles and maternal transmission amplifying the process. All that is now reasonably well understood, as is the truth that the spread can be controlled only by individual precautions to ensure that sexual intercourse does not lead to infection by HIV and that other high-risk behaviour is avoided. The high incidence of HIV infection reported from many African countries is a sign of the end-point which, it is to be hoped, will elsewhere be avoided.

\section{Moving the goalposts}

Disappointment with the World Cup could be simply prevented in future competitions.

Not for the first time, the competition called the World Cup has ended with players and spectators alike complaining of their disappointment. One $\mathrm{BBC}$ commentator was heard to declare that "This hasn't been the best World Cup in the world". But the faults of these competitions, and of the game of football which is their substrate, are well recognized.

This time, the complaint is that too many matches ended with neither side having acquired a score, so that a "result" had to be manufactured, for the convenience of the organizers, by means of a humiliating game of chance in which people kick a football just beyond the reach of a person called a goalkeeper. But that is a superficial issue. It is much more important that the game of football, as at present played, invariably yields such small scores that they cannot possibly be an objective measure of the relative quality of two teams.

Plainly, the game needs radical redesign so as to increase the likelihood that scores different from zero will result. As things are, it is probably fair to represent teams' scoring potential by a Poisson distribution of the form $P(N)=\lambda^{N} \mathrm{e}^{-\lambda} / N$ !, where $P(N)$ is the probability of scoring $N$ in a particular match, when $\lambda$ will be the mean score against comparable opposition. A team's opponents will presumably have a similar distribution, but with a different parameter, say $\mu$. A football game is or should be an experimental test of whether the parameters $\lambda$ and $\mu$ are significantly different. Karl Pearson's $F$ statistic is the appropriate test, but whatever is done, it is inconceivable that there could be a valid test of quality in less than, say, 12 hours play, which would clog all the television channels. It would be simpler to set the goalposts further apart. 